\title{
Research Progress of Traditional Chinese Medicine in Treating Dysphagia After Stroke
}

\author{
Xingxing Shu' ${ }^{1}$, Sina Feng1, Liduo Shen', Ruochen Jiang1, Hui Zhang' \\ ${ }^{1}$ Shaanxi University of Traditional Chinese Medicine, Xianyang 712046, Shaanxi Province, China \\ ${ }^{2}$ Affiliated Hospital of Shaanxi University of Traditional Chinese Medicine, Xianyang 712046, Shaanxi Province, China \\ *Corresponding author: Hui Zhang, 1010179970@qq.com
}

\begin{abstract}
Dysphagia is a common complication after stroke, which does not only affect the quality of life of patients, but also increase the risk of death. Traditional Chinese medicine (TCM) treatment has always played an important role in the clinical treatment of dysphagia after stroke, but its mechanism has not been fully elucidated. Literatures on the use of TCM for the disease in the recent 5 years have been reviewed, and the problems existing in the development and treatment of the disease are discussed in this article, in an attempt to provide new ideas for the treatment of the disease. This paper presents a summary of traditional Chinese medicine treatment for the disease in the past five years, including traditional Chinese medicine compound and acupuncture.
\end{abstract}

Keywords: Stroke; Dysphagia; Traditional Chinese medicine treatment; Review

Publication date: November 2021; Online publication: November 30, 2021

\section{Introduction}

As one of the common complications after stroke, dysphagia, with an incidence of about $20 \%-65 \%{ }^{[1]}$, is mainly manifested as the inability to deliver food or liquid from the mouth to the stomach. Patients with mild symptoms may experience choking on drinking water and difficulty in swallowing, which would affect their desire to eat. Severe symptoms may lead to aspiration pneumonia, dehydration, electrolyte imbalance, malnutrition, and even suffocation, which is life-threatening ${ }^{[2]}$. Dysphagia is a significant factor that contributes to the high mortality of stroke patients ${ }^{[3]}$. The treatment of post-stroke dysphagia in western medicine often focuses on the prevention and treatment of the original disease as well as the rehabilitation of the patients' swallowing function. However, the effect from the rehabilitation is not completely significant, in which there would be some persistent sequelae ${ }^{[4]}$. At present, there is still a lack of highquality evidence-based drugs for dysphagia. Therefore, Western medicine lacks strong evidence to support dysphagia after stroke. The goal of treatment is to improve the amount and type of food consumed, as well as minimize aspiration and related complications. In recent years, non-invasive brain stimulation and nerve electrical stimulation have been widely used in clinical practice, such as neuromuscular electrical stimulation, transcranial direct current stimulation, etc. However, as new instruments, their long-term effects have not been fully verified, and the precise mechanism of neuromuscular electrical stimulation is still unclear; thus, further large-scale research is still needed. For example, the use of transcranial direct current stimulation in the subacute stage is also controversial ${ }^{[5]}$. All these show that the current treatments used in western medicine have certain limitations; hence, it is necessary to explore new treatment methods. 


\section{Chinese medicine compound}

\subsection{Discussing from the perspective of kidney deficiency}

The majority of stroke patients are elderly patients. Elderly people have weak bodies, and their kidneys have been exhausted; in addition, chronic illnesses can damage the kidneys. In view of all these, kidney deficiency is often the main cause. Yellow Emperor's Internal Classic clearly pointed out that the root cause of this disease is kidney deficiency; therefore, the treatment should be based on invigorating the kidneys. In a study ${ }^{[6]}$, a total of 84 patients with post-stroke dysphagia were enrolled and randomly divided into two groups. The therapeutic effects of invigorating the kidney, promoting blood circulation, and swallowing rehabilitation were compared with those of simple swallowing rehabilitation. After 28 days of treatment, the results showed that the total effective rate was $88.10 \%$ in the group that received treatment by invigorating the kidney and promoting blood circulation. It was significantly higher than that of the rehabilitation training group $(69.05 \% ; P<0.05)$. By carrying out the water-swallowing test (WST) and observing the TCM syndrome score of the patients, it was found that the swallowing function in the treatment group improved significantly.

\subsection{Discussing from the perspective of qi deficiency}

In Shou Shi Bao Yuan, it was mentioned, "qi and blood is the basis of the human body." In Puji Fang, it was also mentioned, "Human life is inseparable from qi and blood." This shows the importance of qi and blood to the human body. In stroke patients, lying down for a long time hurts the qi, and the lack of qi would cause weakness; in addition, there is poor blood circulation, which would lead to stasis. In turn, blood stasis produces phlegm, which blocks the throat. In that way, the disease occurs. In another study ${ }^{[7]}$, Buyang Huanwu Decoction was used along with early rehabilitation for swallowing to treat 21 patients with bulbar palsy. The control group received swallowing rehabilitation only. The results revealed that the total effective rate in the observation group was $90.5 \%$, which was significantly higher than that of the control group (42.9\%). This indicates that the use of qi-invigorating drugs plays a very important role in the treatment of post-stroke dysphagia.

\subsection{Discussing from the perspective of phlegm and blood stasis}

In Xue Zhen Lun, it was mentioned, "Long-term blood stasis would cause then phlegm to be turbid." Dysphagia is a common complication after stroke. Phlegm and blood stasis are the main pathogenic factors of stroke, often throughout the stroke, from the beginning to the end. Therefore, the treatment should be based on resolving phlegm and resuscitating, removing blood stasis and promoting blood circulation, as well as removing pathological products in order to achieve the purpose of curing the disease. Su Shujuan and other researchers ${ }^{[8]}$ used the method of removing phlegm and blood stasis in addition to rehabilitation for swallowing to treat 42 patients with post-stroke dysphagia. It was a comparative study between the observation group (Ditan Decoction and Tongqiao Huoxue Decoction combined with swallowing rehabilitation) and the control group (swallowing rehabilitation). After 15 days of treatment, the total effective rate of the observation group was higher than that of the control group; in addition, the swallowing function and the independent-living ability of the patients in the observation group improved significantly. This illustrates the advantages of removing phlegm and blood stasis in the treatment of post-stroke dysphagia.

\section{Acupuncture}

Acupuncture achieves the purpose of dredging channels and collaterals as well as preventing and treating diseases by stimulating acupoints and meridians. Swallowing disorders are closely related to systemic 
visceral dysfunction as well as abnormal qi and blood flow after stroke. These pathological manifestations can be reflected in the meridians. Based on different symptoms, appropriate acupuncture method should be selected and appropriate replenishing and reducing methods should be used. Through this, the body reaches a balanced state of yin and yang, which fundamentally improves the symptoms of dysphagia.

\subsection{Electroacupuncture}

In a study ${ }^{[9]}, 60$ patients were randomly divided into two groups. The patients in the first group were treated with electroacupuncture. By comparing the scores of their swallowing function, it was found that the patients who received the treatment had significant improvement in terms of their swallowing function compared to the other group. It has been proven in an animal experiment that electroacupuncture has a reliable therapeutic effect on dysphagia after stroke, and the best effect is when the output frequency is 2 $\mathrm{Hz}^{[10]}$. This shows that electroacupuncture has a significant effect on improving the swallowing function.

\subsection{Tongue acupuncture}

Li Qingbin and other researchers recruited 60 patients with dysphagia after stroke in a randomized controlled clinical study to determine the clinical efficacy of tongue acupuncture ${ }^{[11]}$. It was found that the total effective rate was $80.00 \%$ and the swallowing function as well as independence-living ability of the patients in the therapy group improved significantly. This shows that tongue acupuncture has a good therapeutic effect in the treatment of dysphagia after stroke.

\subsection{Eye acupuncture}

In a study ${ }^{[12]}$, eye acupuncture was compared with ordinary acupuncture. It was found that both of types of acupunctures have therapeutic effects on patients with dysphagia after stroke, but the effect of eye acupuncture was more prominent. In another study, Zhang Shuxian verified that eye acupuncture can significantly improve the swallowing function of stroke patients, and it is significantly better than simple rehabilitation based on the WST score, Ichiro Fujishima score, and total effective rate ${ }^{[13]}$.

\subsection{Nape acupuncture}

One hundred patients with post-stroke dysphagia were enrolled in a study ${ }^{[14]}$, and they were randomly divided into two groups. The patients in the observation group received nape acupuncture along with rehabilitation (main point: bilateral Fengchi, Yiming, Lianquan, Waijinjin), while those in the control group underwent rehabilitation only. The results showed that the WST score, standardized swallowing assessment (SSA) score, and repeated saliva swallowing test (RSST) score of the nape acupuncture group were higher than those of the control group with obvious improvement, and the difference was statistically significant.

\subsection{Scalp acupuncture}

In a study ${ }^{[15]}$, scalp acupuncture and a swallowing training instrument were used to treat 68 patients with dysphagia after stroke. They were divided into two groups randomly, in which the control group only used the swallowing training instrument, whereas the observation group received scalp acupuncture in addition to the former. By comparing the clinical efficacy between the two groups, it was found that the symptoms experienced by the patients in the scalp acupuncture group, such as choking on water, coughing, and eating difficulties, significantly improved in comparison to the control group. The total effective rate was $90.32 \%$; in addition, the ability of the patients in the observation group to support themselves also improved. 


\section{Discussion}

Through the research on Chinese medicine compound and acupuncture, is has been found that traditional Chinese medicine therapy can effectively improve clinical symptoms, relieve illness, and improve life satisfaction. Moreover, traditional therapy has no risk for dependence and has high patient compliance, which can improve the swallowing function of stroke patients and achieve the purpose of treating both the symptoms and root cause. This shows that traditional therapy has irreplaceable advantages in this field. However, there are still many issues which require further discussion and research, such as the unclear mechanism in using acupuncture as a treatment. The recommendations in the guide are only based on the experience of famous experts ${ }^{[16]}$. Furthermore, there is no unified standard for the screening and evaluation of swallowing in terms of the indicators and tools that can be used ${ }^{[17]}$.Therefore, there is still a need to deeply explore the mechanism of TCM treatment in dysphagia after stroke, in order to gather higher-level evidence support as well as to ensure a more standardized and scientific therapy.

\section{Disclosure statement}

The authors declare that there is no conflict of interest.

\section{References}

[1] Arnold M, Liesirova K, Broeg-Morvay A, et al., 2016, Dysphagia in Acute Stroke: Incidence, Burden and Impact on Clinical Outcome. PLoS One, 11(2): e148424.

[2] Wang A, Liu Y, Dong X, 2015, Reliability and Validity of the Chinese Version Eating Disorders Quality of Life Scale. Journal of Nursing Science, 2015(17): 10-13.

[3] Koestenberger M, Neuwersch S, Hoefner E, et al., 2020, A Pilot Study of Pharyngeal Electrical Stimulation for Orally Intubated ICU Patients with Dysphagia. Neurocrit Care, 32(2): 532-538.

[4] Gu Y, Huan Y, Lv Y, et al., 2006, Method and Effect of Early Comprehensive Rehabilitation for Dysphagia After Stroke. Chinese Journal of Rehabilitation Medicine, 21(1): 71-72.

[5] Jones CA, Colletti CM, Ding MC, 2020, Post-stroke Dysphagia: Recent Insights and Unanswered Questions. Curr Neurol Neurosci Rep, 20(12): 61.

[6] Shan J, Shan X, Yang G, et al., 2020, Clinical Study on the Treatment of Ischaemic Stroke and Swallow Dysfunction with the Method of Kidney-nourishing and Blood-activating. Journal of Liaoning University of Traditional Chinese Medicine, 22(02): 104-107.

[7] Xiong T, 2018, Clinical Observation on Buyang Huanwu Decoction Combined with Early Swallowing Training in the Treatment of Apoplectic Bulbar Paralysis. Guangming Journal of Chinese Medicine, 33(14): 2051-2053.

[8] Su S, Li C, Lin R, et al., 2018, Clinical Efficacy of Ditan Decoction and Tongqiao Huoxue Decoction Combined with Swallowing Function Training on Swallowing Disorders after Cerebral Infarction. Chinese Archives of Traditional Chinese Medicine, 36(7): 1786-1788.

[9] Jin Z, Chen J, Bao D, et al., 2017, Clinical Observation on Treatment of 30 Cases of Dysphagia with Pseudobulbar Palsy after Stroke by Electroacupuncture. Journal of China-Japan Friendship Hospital, 31(2): 173-175.

[10] Yao S, Liu Y, Cui S, et al., 2020, Effect of Different Frequencies of Electroacupuncture on Post-Stroke Dysphagia in Mice. J Mol Neurosci, 70(11): 1871-1879.

[11] Li Q, Huang L, Wang L, et al., 2018, Clinical Study on Functional Training Combined with Lingual Acupuncture in Treating Stroke Dysphagia after Multiple Stroke. Chinese Archives of Traditional 
Chinese Medicine, 36(07): 1686-1689.

[12] Runzhe J, 2017, The Clinical Efficacy of Eye Acupuncture Therapy in the Treatment of Dysphagia After Stroke. Liaoning University of Traditional Chinese Medicine.

[13] Zhang S, 2020, Eye and Belt Acupuncture Rehabilitation Therapy in the Treatment of Dysphagia After Stroke Observation of Curative Effect. Liaoning University of Traditional Chinese Medicine.

[14] Chen F, Liu X, Bao Y, et al., 2018, Clinical Observation of Nape Acupuncture plus Rehabilitation Training in Treating Pseudobulbar Palsy After Cerebral Stroke. Shanghai Journal of Acupuncture and Moxibustion, 37(2): 135-139.

[15] Zhou T, Li L, Feng E, et al., 2017, Clinical Observation of Scalp Acupuncture Combined with Swallowing and Speech Therapeutic Instrument for Treatment of Post-Stroke Dysphagia. Journal of Guangzhou University of Traditional Chinese Medicine, 34(6): 859-863.

[16] Expert Consensus Group for Rehabilitation Evaluation and Treatment of Swallowing Disorders in China, 2018, Expert Consensus on the Evaluation and Treatment of Dysphagia in China (2017 Edition). Chinese Journal of Physical Medicine and Rehabilitation, 40(1): 1-10.

[17] Ge W, Ding L, Murong Z, et al., 2021, Reflections on Screening and Assessment Tools in the Study of Acupuncture in Treating Post-Stroke Dysphagia. Journal of Clinical Acupuncture and Moxibustion, 37(4): 1-6. 